\title{
A wider audience: turning VLBI into a survey instrument
}

\author{
E. Middelberg ${ }^{1, \star}$, A. T. Deller ${ }^{2}$, W. F. Brisken ${ }^{3}$, J. S. Morgan $^{4}$, and R. P. Norris ${ }^{5}$ \\ 1 Astronomisches Institut der Ruhr-Universität Bochum, Universitätsstraße 150, 44801 Bochum, Germany \\ 2 ASTRON, Oude Hoogeveensedijk 4, 7991 PD Dwingeloo, The Netherlands \\ 3 National Radio Astronomy Observatory, PO Box O, Socorro, NM 87801 \\ ${ }^{4}$ International Centre for Radio Astronomy Research, Curtin University, GPO Box U1987, Perth, WA, Australia \\ ${ }^{5}$ CSIRO Astronomy and Space Science, Australia Telescope National Facility, PO Box 76, Epping NSW 1710, Australia
}

Received ..., accepted ...

Published online later

Key words instrumentation: interferometers, techniques: interferometric, methods: observational, surveys

Radio observations using the Very Long Baseline Interferometry (VLBI) technique typically have fields of view of only a few arcseconds, due to the computational problems inherent in imaging larger fields. Furthermore, sensitivity limitations restrict observations to very compact and bright objects, which are few and far between on the sky. Thus, while most branches of observational astronomy can carry out sensitive, wide-field surveys, VLBI observations are limited to targeted observations of carefully selected objects. However, recent advances in technology have made it possible to carry out the computations required to target hundreds of sources simultaneously. Furthermore, sensitivity upgrades have dramatically increased the number of objects accessible to VLBI observations. The combination of these two developments have enhanced the survey capabilities of VLBI observations such that it is now possible to observe (almost) any point in the sky with milli-arcsecond resolution. In this talk I review the development of wide-field VLBI, which has made significant progress over the last three years.

(C) 0000 WILEY-VCH Verlag GmbH \& Co. KGaA, Weinheim

\section{Introduction}

Since the invention of the VLBI technique in the 1960s, observations using it have almost exclusively been limited to the study of carefully selected, small samples of objects. There are two fundamental reasons which have led to this situation. First, the observing bandwidth is restricted by the need to record the raw data on tape or disk and then replay and process the data afterwards on a reasonable timescale. Since the sensitivity of an interferometer is linked to the bandwidth of the observations (i.e., the number of measurements being made), this limitation directly affected the sensitivity. Connected-element interferometers such as the VLA ${ }^{1}$ and ATCA $^{2}$ are not affected by this, since there is no need to store the raw antenna signals before the correlation is carried out. Second, the field of view of a radio interferometer is limited by the spectral and temporal resolution with which the data are correlated (which can also be expressed as the channel width, $\Delta \nu$, and integration time, $\Delta \tau$, over which the data are averaged). In an oftenused analogy the limitation arising from bandwidth averaging can be compared to chromatic aberration in a lens, and the limitation from time averaging to motion blur in a photograph when the exposure time has been too long. Whilst both effects also affect connected-element interfer-

\footnotetext{
^ Corresponding author: e-mail: middelberg@astro.rub.de

1 Very Large Array

2 Australia Telescope Compact Array
}

ometers, the long baselines and consequently high spatial resolution of VLBI observations limit the field of view to around one arcsecond. Therefore traditional VLBI observations are helplessly unsuitable for surveys of large fractions of the sky.

\section{Bandwidth and time averaging smearing}

In a radio interferometer image suffering from too low spectral resolution and/or too long integration times, radio sources away from the field centre get blurred and extended. These two effects are called bandwidth and time smearing respectively. The magnitude of the effect can be estimated following Thompson et al.(2001), chap. 6.4. Here, I provide a qualitative explanation for the effects.

A radio interferometer is sensitive to the spatial coherence of the electromagnetic radiation received at two antennas. One can show that this spatial coherence function is, within certain limits, equivalent to the Fourier transform of the sky brightness distribution (see Clark 1999 for an introduction). At any one time, a single baseline makes a measurement of the complex Fourier plane, at a location given by the length and relative orientation of the baseline with respect to the direction to the source. Since the sky plane has axes with units of angles, the Fourier plane, called $(u, v)$ plane by radio astronomers, also must have dimensionless units, which in this case correspond to wavelengths. The 

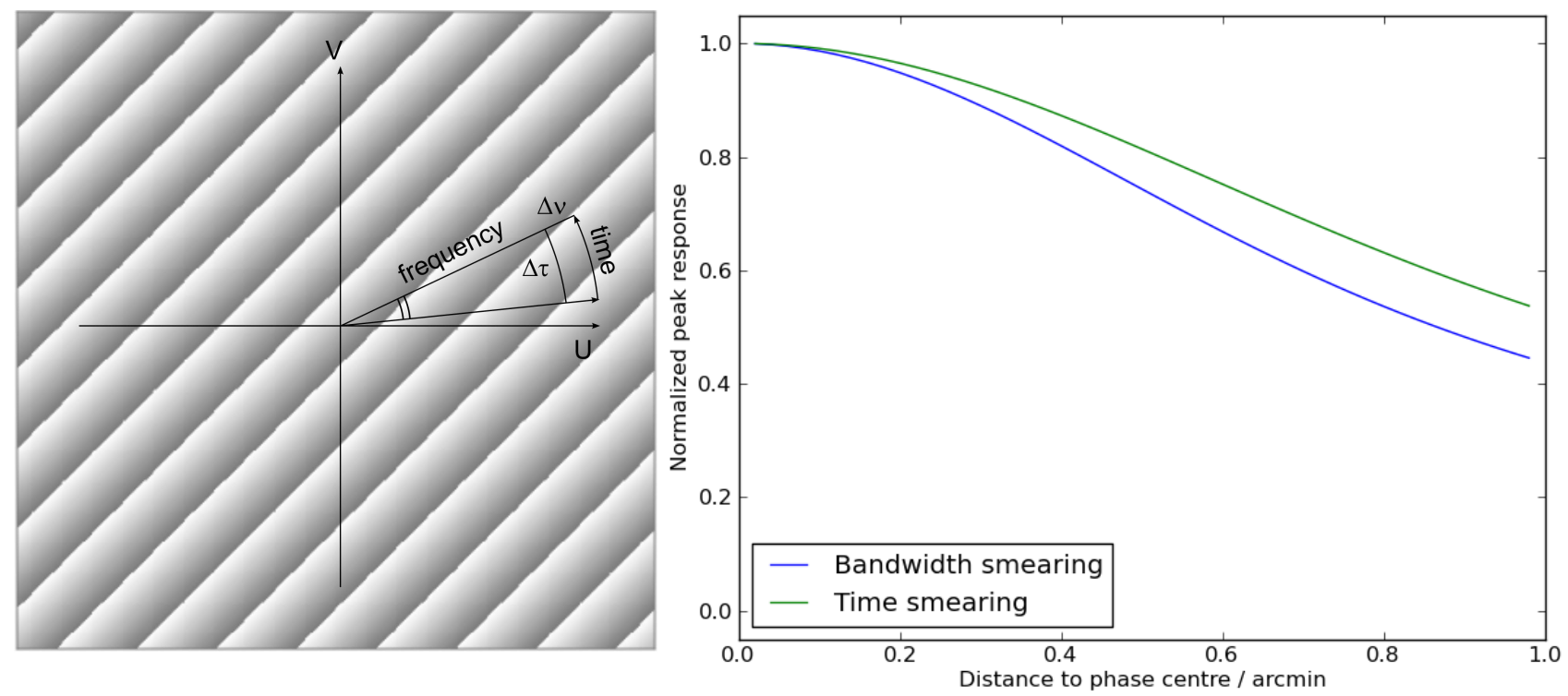

Fig. 1 Illustration of bandwidth and time smearing. Left panel: Shown is the $(u, v)$ plane in which an interferometer baseline makes a measurement. The sinusoidal phase variations of a point source offset from the field centre are indicated by the background greyscale image. The frequency of the phase variations is proportional to the distance of a source to the field centre, and its orientation by its angle in the $(u, v)$ plane. The ratio of the observing frequency, $\nu$, and the bandwidth, $\Delta \nu$, is independent of baseline length, and therefore the fraction covered by $\Delta \nu$ along the baseline vector increases with the baseline length. The area covered by the baseline vector within the integration time, $\Delta \tau$, is caused by the angular velocity of the earth's rotation and therefore also is a function of distance to the centre of the $(u, v)$ plane. The Fourier transform of the sky brightness does not change much on short baselines, close to the field centre, and so the measurement is coherent in frequency and time. On longer baselines, however, the measurement is integrated over an area in which the phase of the Fourier transform change by more than one radian, and therefore coherence is lost. Right panel: the decrease of sensitivity caused by bandwidth and time smearing, estimated using the approximations given by Thompson et al. (2001). The calculation has been carried out for conditions typical of traditional VLBI observations: a frequency of $1.4 \mathrm{GHz}$, a channel width of $500 \mathrm{kHz}$, a baseline length of $5000 \mathrm{~km}$ and an integration time of $4 \mathrm{~s}$. At a distance of 0.4 arcmin ( 24 arcsec) both bandwidth and time smearing alone cause the sensitivity to decrease to about 0.84 , causing a combined loss of $0.84^{2}=0.7$. To compensate for this sensitivity loss the total observing time during the observations would need to be approximately doubled.

axes of the Fourier plane are chosen such that $v$ points towards the coordinate north pole.

Since a measurement requires to collect energy, it must be carried out using a finite bandwidth and a finite integration time. Thus a measurement carried out by a baseline does not correspond to a point in the Fourier plane, but to a small area along an arc traced out by the baseline in the $(u, v)$ plane (caused by the rotation of the earth during the integration). In the case of a point source offset from the field centre the phase in the Fourier plane changes as illustrated in Fig. 11 (the amplitude is constant across the $(u, v)$ plane for point sources). Consequently, longer baselines measure Fourier components farther away from the field centre, and potentially integrate over an area so large that the phase of the Fourier transform changes, and so coherence is lost. The frequency of the sinusoidal phase variations indicated in Fig. 1 is proportional to the distance of the point source to the field centre. Hence shorter baselines can image larger fields, and this is the fundamental reason for the tiny fields of view of traditional VLBI observations.
An example for the magnitude of the effect is given in the right panel of Fig. 1

\section{Enabling wide-field VLBI with software correlators}

From the illustration in the left panel of Fig. 1 one can (correctly) conclude that to preserve coherence on long baselines for wide fields of view it is sufficient to decrease $\Delta \nu$ and $\Delta \tau$. However, increasing the field of view of a VLBI array to a dimension which is comparable to the field of view of the interferometer elements (of order $\lambda / D$, or 30 arcmin) requires to produce data with $\mathrm{kHz}$ resolution, and a temporal resolution of order tens of ms. This requirement is far in excess of what had been available at correlators until very recently.

Fortunately, the power of commodity hardware computers had increased by 2005 to such a degree that the correlation of interferometer data using software on computer 
clusters became feasible. Correlation is what is known as an "embarrassingly parallel" problem, since it can be divided up in frequency, time, and baseline, and no communication between the correlator processes is required. In 2007 a software correlator was presented by Deller et al. (2007). Its main features are almost unlimited spectral and temporal resolution, superb scalability, and speed, since it makes use of specific optimisations in Intel CPUs. Meanwhile a deployment of DiFX, called VLBA-DiFX, has replaced the hardware correlator of the VLBA 3

It was therefore feasible by 2006 to start a project to image all known radio sources in the CDFS ${ }^{4}$ with the VLBA. The observations were carried out in 2007, and the telescope data were shipped to the MPIfR ${ }^{5}$ in Bonn, where a computer cluster was available for correlation.

\section{Wide-field VLBI observations of the CDFS}

When this project was initiated it was not clear in what way the DiFX software correlator would be used to produce the desired data. It was nevertheless attempted to select a field for the observations with good complementary data to enhance the interpretative value of the results. The CDFS is arguably the best-studied area in the sky and was therefore chosen, despite its declination of $-27^{\circ}$ which makes it a challenging target for the VLBA. The project is described in full detail in Middelberg et al. (2011), and we give here a condensed summary.

\subsection{Observations, correlation and calibration}

The CDFS was observed on 3 July 2007 with the VLBA at $1383 \mathrm{MHz}$. An overview of the field is given in Fig. 3 The low declination of the field resulted in an elapsed time of the observations of only $9 \mathrm{~h}$, and the expected sensitivity was $32 \mu \mathrm{Jy}_{\text {beam }}{ }^{-1}$. Two effects increase this estimate: the need to self-calibrate on a suitably bright target in the field resulted in a loss of about $40 \%$ of the data, and the low declination caused the antenna system temperatures to increase of the order of $30 \%$ to $40 \%$. The final sensitivity in the centre of the field was found to be $55 \mu \mathrm{Jy}_{\text {beam }}{ }^{-1}$.

To keep the amplitudes within $5 \%$ of their true values, one would have to use a channel width of $4 \mathrm{kHz}$ and $50 \mathrm{~ms}$ integrations. Such a correlation would result in around $3 \mathrm{~TB}$ of visibility data, which would be very difficult to manage even on large general-purpose computers. Fortunately, the DiFX software correlator has recently been extended to support the simultaneous correlation of multiple field centres. This mode is analogous to what has previously been known as "multi-pass correlations" on hardware correlators, but in DiFX it is implemented efficiently and supports several hundred phase centres at a comparatively small computational cost. The details of this mode are described in Deller et al.

\footnotetext{
3 Very Long Baseline Array

4 Chandra Deep Field South

5 Max-Planck Institut für Radioastronomie
}

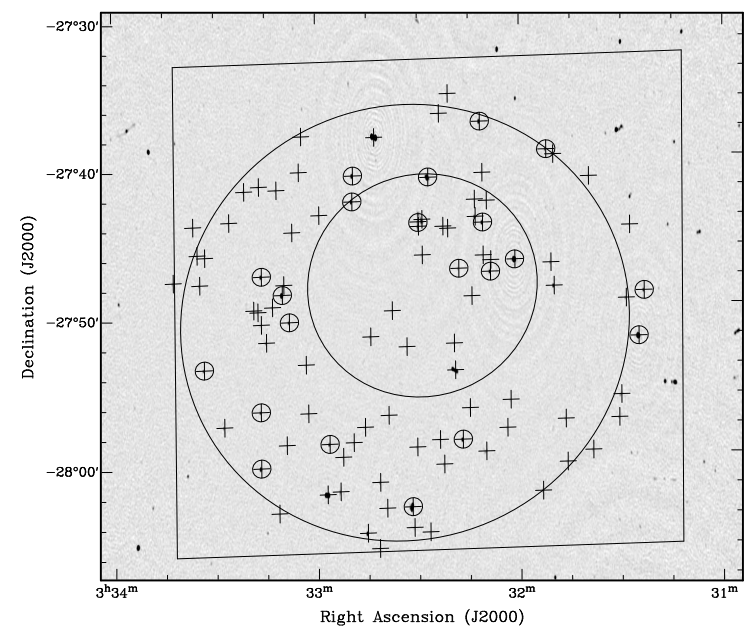

Fig. 3 An overview of the observed area. The background image is a radio image of the CDFS made with the Australia Telescope Compact Array (Norris et al. 2006). The rms of the image is around $20 \mu \mathrm{Jy}_{\text {beam }}{ }^{-1}$, and the faintest sources have flux densities of $100 \mu \mathrm{Jy}$. The square indicates the eCDFS area observed with the Chandra satellite by Lehmer et al. (2005) with an integration time of $240 \mathrm{ks}$; the large circle indicates a typical VLBA antenna's primary beam size at the half-power level at $1.4 \mathrm{GHz}$; and the medium circle is the region covered uniformly with a $2 \mathrm{Ms}$ exposure with Chandra. Crosses are drawn at the locations of the 96 targets taken from Norris et al. (2006) and small circles around those targets which were detected with the VLBA.

(2011). The idea is to place one phase centre on each known radio source. The efficiency of the DiFX implementation arises from the fact that the computationally expensive FFT of the incoming antenna data needs to be computed only once, and the subsequent computations for each phase centre only require complex multiplications at a rate of around 100 per second. This procedure results in one normal-sized VLBI data set per phase centre. The field of view of each data set is small (of order 10 arcsec), but the source coordinates are sufficiently well known that bandwidth and time averaging effects are not an issue.

Amplitude calibration was carried out using $T_{\text {sys }}$ measurements and known gain curves. Initial phase calibration was done using regular interleaved scans on a nearby calibrator source. However, the low declination of the field caused significant incoherence of the data and required selfcalibration. Fortunately one target was detected with sufficient SNR for self-calibration, although visibilities on long baselines had poor SNR and caused a loss of around $40 \%$ of the data which could not be calibrated. Since the phase response of a VLBA antenna is constant within the primary beam, and since the geometric delays had been compensated at the correlation stage, the amplitude, phase, and delay correction could simply be copied from one target 

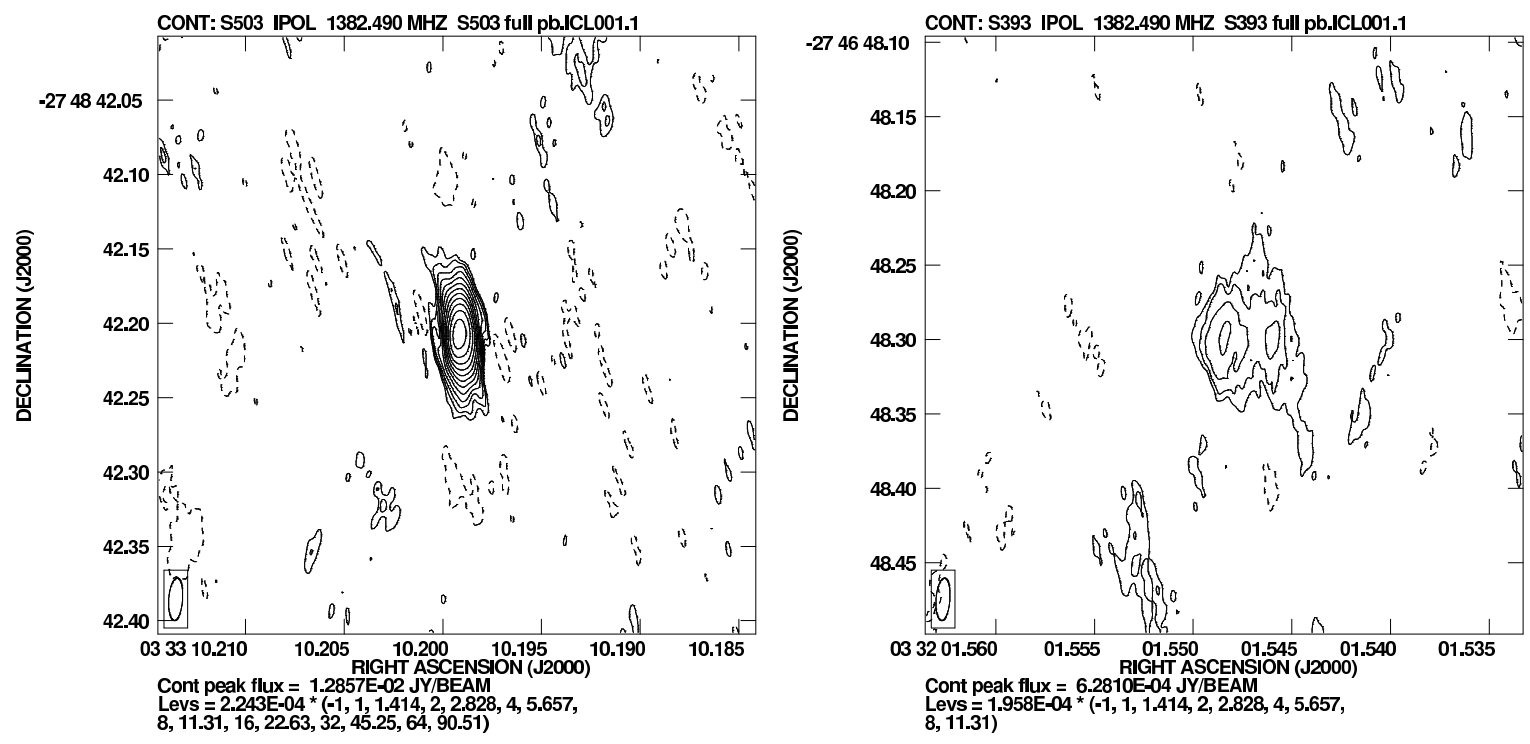

Fig. 2 Contour plots of two sources from the VLBI survey of the CDFS. Contours start at three times the image rms level and increase by factors of $\sqrt{2}$. Both images suffer from calibration artifacts, arising from the low declination of the field. Left panel: Contour plot of S503, the strongest detected target, which served as an in-beam calibrator to improve the coherence of the data after initial phase calibration. The image noise is $0.75 \mathrm{mJy} \mathrm{beam}^{-1}$ and the integrated flux density is $21.2 \mathrm{mJy}$. Right panel: Contour plot of S393, one of the few detected targets with sub-structure on mas scales. The image noise is $0.65 \mathrm{mJy}$ beam $^{-1}$ and the integrated flux density is $24.7 \mathrm{mJy}$.

source to another, and images could be formed. However, another correction is needed to compensate for the amplitude response arising from primary beam attenuation, which reduces the apparent flux density of a source by up to $50 \%$.

Unlike in compact-array interferometry, where the primary beam correction is carried out in the image plane, we corrected for primary beam attenuation by calculating visibility gains. This is possible only because the fields of view are very small in our observations and so the attenuation due to the primary beam does not vary significantly across the image. We have developed a correction scheme which uses measured beam widths and the relative orientations of the antennas and targets in the telescopes' field of views to compensate for the primary beam attenuation. As a result we were able to produce calibrated $1.4 \mathrm{GHz}$ VLBI images for 96 targets from a single observing run with the VLBA.

\subsection{Results}

Out of the 96 targets, 20 were confidently detected (peak flux density exceeding 6 times the local noise), and one more target was detected with lower significance. Two examples are shown in Fig. 2. The large amount of ancillary data in the CDFS allowed us to supply our observations with substantial multi-wavelength data. In particular, a redshift was available for almost all sources, and the entire field had been covered with deep X-ray observations as part of the CDFS and the eCDFS ("extended" CDFS) surveys. It was found that despite the large amount of data, seven of the detected targets had not previously been identified as harbouring an active nucleus. Since our VLBI detections required brightness temperatures in excess of $4.2 \times 10^{5} \mathrm{~K}$ to make a detection, each of these sources could readily be identified as containing an AGN (using the available redshifts for the additional argument that the luminosity must exceed that of galactic, high-brightness temperature sources). The multiwavelength data of one source, S423, indicates that it is a starburst galaxy, however, the VLBI detection clearly shows that it also contains an AGN. Combined sources such as this are rare, but they are thought to be more frequent at high redshifts, and to identify them in large numbers requires VLBI survey techniques. A marginal detection was made in S443, the location of which at the edge of a spiral galaxy (visible in a Hubble Space Telescope image) suggests that this could be a radio supernova.

\section{Wide-field VLBI observations of the Lockman Hole/East region}

The observations described in the previous section were mainly intended to explore the capabilities of the DiFX correlator in wide-field VLBI observations, and to develop a primary beam calibration scheme. For larger surveys, as they are commonly carried out in mosaic mode with compact interferometers, however, additional techniques were needed. For example, only the fortuitous circumstance of having a suitably bright target within the primary beam allowed us to calibrate (most of) the data. In a survey of a larger region with many overlapping pointings, not many such sources can be expected, preventing calibration. A project was therefore initiated to take wide-field VLBI one 
step further, and the Lockman Hole/East region was chosen as the target. This field had been observed with the VLA, with a sensitivity of less than $10 \mu \mathrm{Jy}_{\text {beam }}{ }^{-1}$, resulting in the detection of 1450 radio sources. Three full synthesis observations of $12 \mathrm{~h}$ duration were needed to cover the area. Since the sensitivity of the VLBA reaches around $24 \mu \mathrm{Jy}$ beam $^{-1}$ in this time, the overlap of the three pointings resulted in a sensitivity of around $18 \mu \mathrm{Jy}_{\text {beam }}{ }^{-1}$, and all 532 sources with integrated flux densities of more than $100 \mu \mathrm{Jy}$ were targeted. An overview of this field is shown in Fig. 4 .

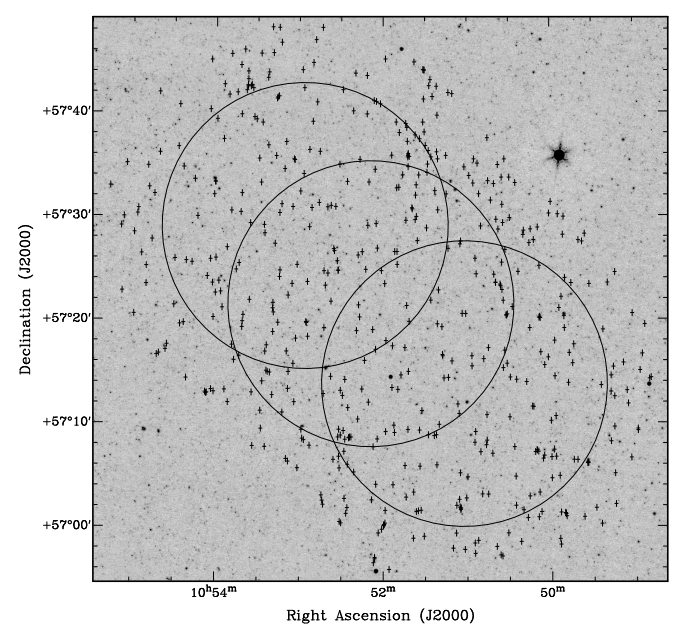

Fig. 4 Overview of the VLBA observations of the Lockman Hole/East. Shown is a Spitzer $3.6 \mu$ m greyscale image, circles denote the typical FWHM circle of a VLBA antenna at $1.4 \mathrm{GHz}$, and crosses indicate the locations of the 532 targets.

\subsection{Observations and calibration}

In 2010, three overlapping pointings were observed. Each pointing contained around 320 suitable targets, and each of these targets were used for correlation using the new DiFX multi-phase centre mode. Many targets were present in more than one pointing, and only the combination of these pointings would result in the full available sensitivity.

After initial calibration no targets were found sufficiently bright for self-calibration, and a calibration scheme had to be developed to use the signal from many faint, separate sources for self-calibration. This scheme works as follows:
- Phase-referenced images were made for all targets, and the images were searched for emission. Typically around 30 sources were found with a SNR of more than 7, with the brightest reaching an SNR of almost 100. Since the data are later combined using weights which are proportional to the square of the SNR, a source with $\mathrm{SNR}=10$ contributes only $1 / 100$ of the combined signal compared to a source with SNR=100. Therefore only the brightest 10 sources or so were used in the following steps.

- The individual data sets were divided by the Clean model obtained during imaging. This results in data sets each showing a $1 \mathrm{Jy}$ point source in the field centre. It is worth noting that in this process the data weights are modified according to the amplitude of the input model. Since at this stage no primary beam correction had been carried out, the noise in all images is the same, and so the measured amplitude is directly proportional to the SNR in the image. Hence this process conveniently takes care of proper weighting when the data are combined.

- The source coordinates in the data set headers were set to the same value. This results in data sets with a $1 \mathrm{Jy}$ source at the same coordinates, even though the signal in the data comes from separate sources. For each baseline, time, and frequency the combined data set now contains multiple measurements of a point source.

- Self-calibration was used with a $1 \mathrm{Jy}$ point source model to improve the coherence of the combined data set. The phase corrections derived in this process were then copied to all original data sets, and improved images could be made. The improvement in image quality is illustrated in Fig. 5 .

As a result noise-limited images of all targets from each epoch were obtained. A fundamental consequence of this procedure, however, should not go unnoticed: it substantially lowers the sensitivity threshold for self-calibration. Therefore, given sufficient instantaneous sensitivity and large primary fields of view, most of the sky is accessible to VLBI observations at low $\mathrm{GHz}$ frequencies. All it takes to calibrate the data is to add a few (tens) of phase centres towards known sources, taken for example from the NVSS survey. These data can then be used in multi-source selfcalibration and the phase correction can be applied to the target source data.

Another important step was to combine the data from the same target observed during different epochs. Since the positions of the targets were essentially referenced to the position of the calibrator used during the observations, it is expected that a systematic offset can be found between epochs. This offset was determined by measuring the peak positions of several bright, detected targets in each epoch and calculating the average of the position differences. The systematic offsets were found to be small, of order less than 1 mas (approximately $1 / 10$ of a synthesized beam width).

\footnotetext{
${ }^{6}$ National Radio Astronomy Observatory Very Large Array Sky Survey
} 

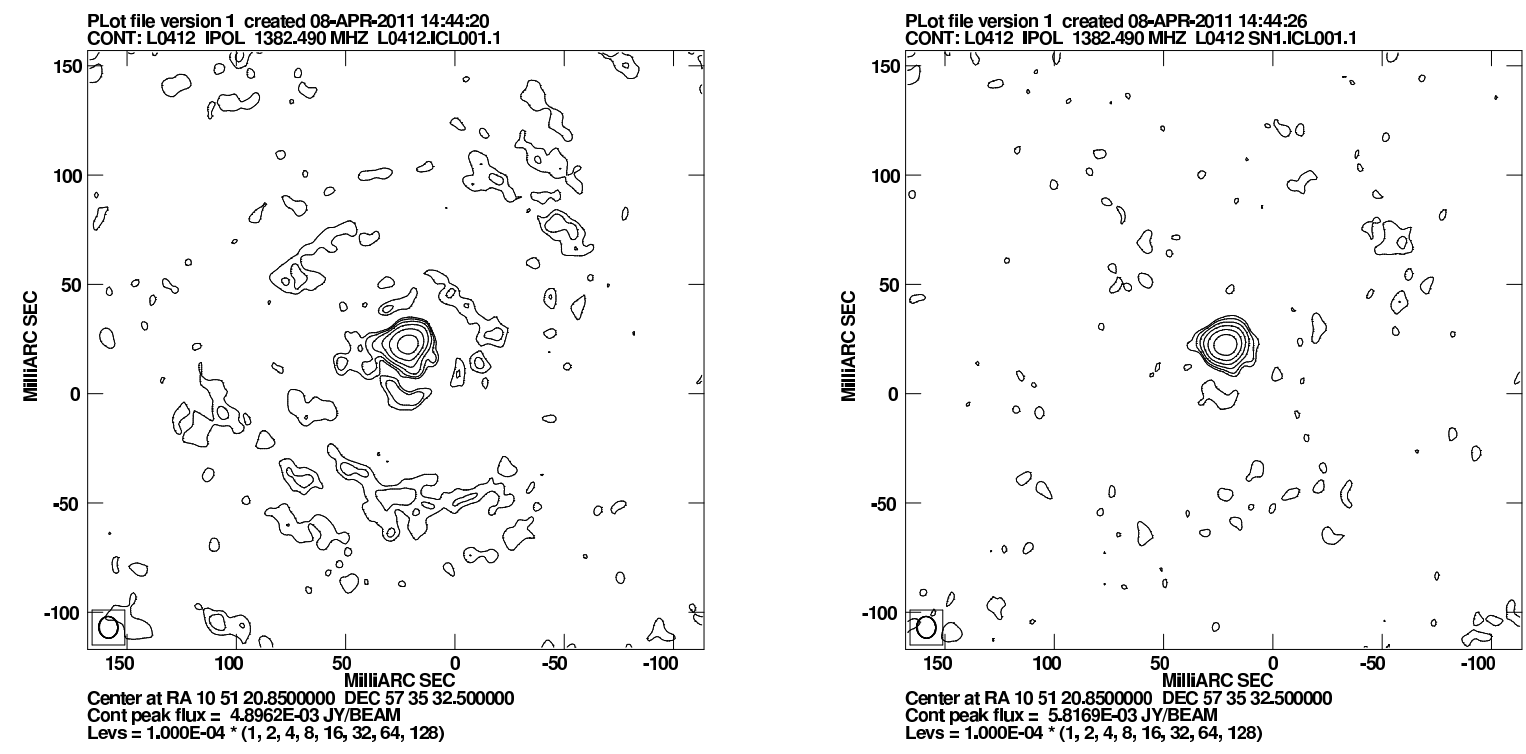

Fig. 5 Contour plot of the radio source L0412 before (left) and after (right) multi-source self-calibration has been applied. Contours are drawn at $0.1 \mathrm{mJy} \times(1,2,4, \ldots)$. The signal-to-noise ratio was improved from from 72 to 115 . The peak flux density has increased significantly, the noise has dropped, and image artifacts are much reduced.

The data were then phase-shifted to compensate for this offset. Subsequently, primary beam calibration as described in the previous section was performed and the data for each source, taken from the different epochs, were combined and imaged.

\subsection{Results}

The calibration of the data had not been finished at the time of writing. In particular it was noticed that the primary beam correction scheme was unsuitable when sources are located outside the primary beam (which is the case for a substantial number of targets, see Fig. 4.) More accurate beam measurements are underway, and calibration is expected to be finished by the end of 2011. A preliminary analysis, however, indicates that around 70 targets have been detected, a fraction smaller than in the CDFS. One effect leading to this result probably is that the sources are generally fainter, and that therefore more starburst galaxies are contained in the sample, which leads to fewer detections.

\section{Conclusions}

Wide-field VLBI observations are now practical and relatively easy to carry out. The new multi-phase centre mode of the DiFX software correlator allows one to observe a large number of sources simultaneously in a single observation, and the multi-source self-calibration strategy we present here enables noise-limited calibration of these data. We point out that wide-field VLBI observations have a number of applications outside the study of galaxy populations in sensitive surveys: a sample of (relatively) faint background sources can serve as a reference in astrometric observations to measure proper motions and parallaxes; and observations of faint targets are no longer limited by the accuracy of phase corrections derived from interleaved calibrator observations. The full potential of wide-field VLBI observations, however, is expected to be reached in even more sensitive observations of large fields such as the COSMOS survey, or in less sensitive observations of very large areas (tens or hundreds of square degrees).

Acknowledgements. 


\section{References}

Clark, B. G. 1999, in ASP Conf. Ser. 180: Synthesis Imaging in Radio Astronomy II, ed. G. B. Taylor, C. L. Carilli, \& R. A. Perley, 1

Deller, A. T., Brisken, W. F., Phillips, C. J., et al. 2011, PASP, 123, 275

Deller, A. T., Tingay, S. J., Bailes, M., \& West, C. 2007, PASP, 119,318

Garrett, M. A., Wrobel, J. M., \& Morganti, R. 2005, ApJ, 619, 105

Lehmer, B. D., Brandt, W. N., Alexander, D. M., et al. 2005, ApJS, 161,21

Lenc, E., Garrett, M. A., Wucknitz, O., Anderson, J. M., \& Tingay, S. J. 2008, ApJ, 673, 78

Middelberg, E., Deller, A., Morgan, J., et al. 2011, A\&A, 526, A74

Norris, R. P., Afonso, J., Appleton, P. N., et al. 2006, AJ, 132, 2409

Thompson, A. R., Moran, J. M., \& Swenson, G. W. 2001, Interferometry and synthesis in radio astronomy (New York, WileyInterscience, 692 p., 2nd ed.) 